\title{
Aplicação da tomografia computadorizada de feixe cônico no diagnóstico de fraturas radiculares
}

\section{Application cone beam computed tomography in Diagnosis the Root Fractures}

\author{
Ana Márcia Viana Wanzeler \\ Especialista em Radiologia e Imaginologia pela UFRJ, Aluna do Mestrado em Clínicas \\ Odontológicas com ênfase em Radiologia pela Universidade Federal do Pará. Belém, PA, Brasil. \\ SÂMILA GonÇALVES BARRA \\ Especialista em Radiologia e Imaginologia pela UFRJ, Aluna do Mestrado em Clínicas \\ Odontológicas com ênfase em Radiologia pela Pontifícia Universidade \\ Católica de Minas Gerais. Belo Horizonte, MG, Brasil. \\ FÁbIO RIBEIRO Guedes \\ Doutor em Radiologia pela Unicamp, professor adjunto, departamento de patologia e \\ diagnóstico oral da Universidade federal do Rio de Janeiro. Rio de Janeiro, RJ, Brasil.
}

\section{RESUMO}

Introdução: A TCFC (Tomografia Computadorizada de Feixe Cônico) está sendo muito utilizada para análises de fraturas radiculares, visto que entre todos os exames de imagens disponíveis é o único exame que possibilita a visualização das três dimensões do objeto. Objetivo: Este artigo fornece uma revisão de literatura da aplicabilidade da Tomografia computadorizada por feixe cônico para a avaliação diagnóstica de fraturas radiculares. Material e Método: A pesquisa foi realizada nas bases de dados BVS e PubMed. As referencias selecionadas foram publicadas nos idiomas inglês e português, sendo empregado os seguintes descritores: "root fracture; cone beam computed tomography; endodontics e diagnosis" no período de 2003 a 2015. Resultados: $6^{\circ}$ artigos foram encontrados no total e de acordo com os critérios de inclusão e exclusão, 27 manuscritos foram selecionados. Conclusão: Diante dos trabalhos apresentados e discutidos, conclui-se que existem vantagens na utilização da tomografia computadorizada de feixe cônico pela possibilidade de uma análise tridimensional que pode nos favorecer um melhor diagnóstico e, por conseguinte, em um melhor planejamento e prognóstico em endodontia.

Palavras-chaves: Tomografia Computadorizada de Feixe Cônico; Endodontia; Diagnóstico; Raiz dentária.

\begin{abstract}
Introduction: The CBCT is being widely used for analysis of root fractures, as among all the tests of frames is the only exam that enables the visualization of three-dimensional object. Objective: This article provides a literature review of the applicability of computed tomography cone beam for diagnostic evaluation of root fracture, with demonstration of some imaging tests. Methods: Surveys were conducted in the VHL databases and PubMed by selecting references published in Portuguese and English, using the following descriptors: "root fracture; cone beam computed tomography; endodontics and diagnosis" from 2003 a 2015. Results: We found 60 articles, and according to the inclusion and exclusion criteria, were selected 27 manuscripts. Conclusion: The works presented and discussed, it is concluded that there are advantages in the use of computed tomography cone beam by the possibility of a three-dimensional analysis that can encourage better diagnosis and therefore in better planning and prognosis in endodontics.
\end{abstract}

Keywords: Cone-Beam Computed Tomography; Endodontics; Diagnosis; Tooth Root. 


\section{INTRODUÇÃO}

As fraturas dentárias são lesões que apresentam ruptura do tecido dentário caracterizada pela presença de uma linha de descontinuidade que pode apresentar-se em diversas orientações. ${ }^{1}$ As fraturas longitudinais podem ser classificadas em relação à sua localização, direção e sua extensão, que são classificadas em quatro tipos: 1) fratura de cúspide, que se inicia na cúspide e se estende até a região cervical, podendo atingir esmalte, dentina e polpa; 2) trinca dentária, iniciandose na coroa e se estendendo até a raiz; 3 ) fratura com separação dentária, em que há o envolvimento de todos os tecidos dentários e que se estende mais apicalmente; e 4) fratura radicular vertical, que ocorre apenas na raiz e apresenta menor grau de sinais e sintomas. ${ }^{2}$

O diagnóstico de fraturas radiculares é um grande desafio durante os exames iniciais, imediatamente após o trauma, já que os sintomas são variáveis e inespecíficos. ${ }^{3}$ Em decorrência dessa dificuldade de se obter o diagnóstico preciso, muitas vezes são lançados meios de visualização direta da linha de fratura, que pode ser realizada com a remoção da restauração, do núcleo e/ou guta-percha pela coroa ou pela raiz, com acesso cirúrgico, vista com o auxílio de microscópio óptico, de transiluminação, coloração ou introdução de uma cunha para a separação dos fragmentos. Entretanto, tais métodos são considerados invasivos. ${ }^{4,5}$

Outra forma de diagnóstico indicado seriam os exames por imagem, que são métodos de caráter não invasivo e frequentemente utilizados na prática clínica. ${ }^{5} \mathrm{~A}$ radiografia intrabucal convencional, por ser um dos métodos auxiliares mais acessíveis, é um dos mais usados. Os achados radiográficos mais comuns são radiolucências apicais e laterais inespecíficas, especialmente quando os fragmentos estão justapostos e sem separação por edema ou tecido de granulação. No entanto, devido à sua natureza bidimensional, há sobreposição das estruturas adjacentes que interferem na visualização direta da linha de fratura, limitando a sensibilidade da técnica para a detecção desse tipo de patologia. A análise é ainda prejudicada se a direção do feixe de raios $X$ não estiver paralela ao plano de fratura. A presença de artefatos nas imagens, devido ao preenchimento de materiais radiopacos intrarradiculares, é outro fator que pode também mascarar a visualização..$^{6,7}$

Com o advento da Tomografia Computadorizada por Feixe Cônico (TCFC) e softwares específicos para a odontologia, ferramentas avançadas para a manipulação das imagens estão à disposição para os cirurgiões-dentistas de todas as especialidades odontológicas. Imagens em terceira dimensão (3D), mensurações precisas, imagens sem distorção ou sobreposição e novos planos de visualização para facilitar o diagnóstico estão disponíveis no dia a dia da clínica odontológica. A imagem gerada pelos tomógrafos de feixe cônico tem sido foco de inúmeros estudos para o seu melhor entendimento e utilização dos recursos. ${ }^{8}$

As aplicações das imagens geradas nos tomógrafos por feixe cônico dentro das especialidades odontológicas são inúmeras, aplicadas nas diversas áreas da odontologia, como: Implantodontia, Ortodontia, Cirurgia oral menor, Bucomaxilofacial, Periodontia, Endodontia, no estudo das estruturas ósseas das Articulações Temporomandibulares (ATM). ${ }^{9}$ Esse tipo de tecnologia permite a criação de protótipos, a realização de simulações cirúrgicas, análises cefalométricas e uma série de trabalhos sem a necessidade da presença física do paciente, oferecendo ao profissional a possibilidade de realizar um melhor diagnóstico, bem como selecionar a terapia mais indicada para o caso. ${ }^{10}$

Porém, apesar das múltiplas vantagens apresentadas pelas imagens adquiridas nos tomógrafos por feixe cônico, algumas 
limitações surgem frente aos estudos e à aplicação clínica das mesmas. Nesse aspecto, um problema seguidamente evidenciado é a formação de artefatos de imagem. Tal problema surge devido a inúmeros fatores, como quilovoltagem e miliamperagem baixas utilizadas pelos tomógrafos por feixe cônico na aquisição das imagens, gerando uma maior quantidade de radiação dissipada frente a elementos de grande densidade, fazendo que, mesmo após a reconstrução das imagens básicas pelos softwares, onde se consegue filtrar alguns artefatos gerados, ainda apareçam alterações significativas que, em muitos casos, comprometem a qualidade diagnóstica do exame. Além disso, problemas na regulagem do aparelho, movimentação do paciente durante a tomada tomográfica e limitações dos algoritmos usados na formação da imagem adquirida são fatores que influenciam e podem determinar alterações na imagem pela geração de artefatos. ${ }^{11,12,13}$

Este artigo propôs realizar uma revisão de literatura sobre a capacidade diagnóstica da tomografia computadorizada por feixe cônico para a detecção de fraturas radiculares e discutir a interferência dos artefatos de imagens que são gerados nos exames, no período de 2003 a 2015 nas bases de dado SciELO e Pudmed, empregando os descritores "Cone-Beam Computed Tomography (CBCT), endodontic, three-dimensional images". Foram encontradas 39 referências, selecionadas 27 e excluídas 12, utilizando como critérios de inclusão que estivessem na língua inglesa ou portuguesa e publicadas entre os anos de 2003 a 2015. Os critérios de exclusão foram: artigos publicados anteriores a 2003 e dissertações de graduação, mestrado ou doutorado.

\section{Metodologia}

Este estudo consistiu em uma revisão da literatura que tem por objetivo elucidar a aplicabilidade da tomografia computadorizada de feixe cônico para a avaliação diagnóstica de fraturas radiculares. Trata-se de estudo com caráter descritivo, que compreendeu o levantamento de referencial realizado nas bases de dados eletrônicas, nacionais e internacionais, BVS e PubMed, selecionandose referências publicadas nos idiomas português e inglês, utilizando os seguintes descritores: "root fracture; cone beam computed tomography; endodontics and diagnosis" no período de 2003 a 2015. Os artigos identificados pela estratégia de busca foram avaliados, de forma independente e cega, por dois pesquisadores (autores), obedecendo rigorosamente aos critérios de inclusão. A seleção dos artigos foi feita a partir dos títulos e resumos, e a busca foi realizada durante sete meses corridos.

Foram selecionados trabalhos que estivessem dentro dos seguintes critérios: textos disponíveis na íntegra; período de publicação entre os anos 2003 a 2015; idioma (português e inglês). Não houve restrições sobre o tipo de estudo e amostra. Foram excluídos os estudos que não obedeceram aos critérios de inclusão supracitados e estudos encontrados em mais de uma base de dados.

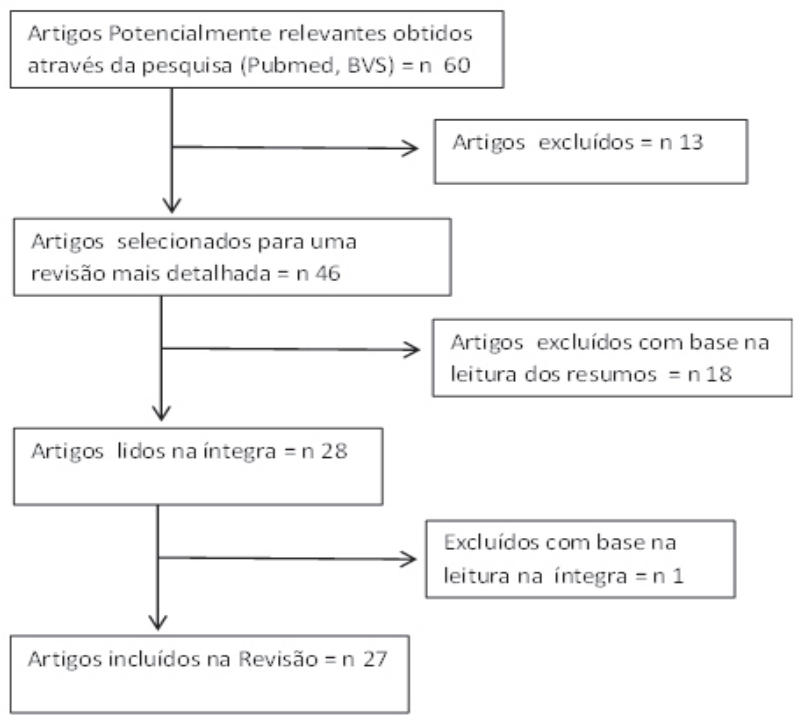

Figura 1: Fluxograma metodológico 


\section{REVISÃO DE LITERATURA}

Em endodontia, as aplicações da TCFC incluem diagnóstico precoce de lesões periapicais, identificação e localização de reabsorções interna e externa, observação de anatomia radicular, detecção de fraturas radiculares e planejamento cirúrgico. ${ }^{13}$

Existem dois tipos de tomografia computadorizada, a tomografia computadorizada fan bean (TCFB), conhecida como tomografia médica, e a tomografia computadorizada por feixe cônico (TCFC), também chamada de tomografia odontológica. As duas tomografias podem obter imagens em cortes da região dentomaxilofacial. ${ }^{14}$

A TCFB utiliza um feixe de raios $X$ em forma de leque, e a TCFC, dedicada ao complexo dentomaxilofacial, utiliza um feixe de raios $X$ em forma de cone, onde captura todas as estruturas ósseas em um só volume, gerando imagens precisas e uma redução significativa de artefatos metálicos. ${ }^{15}$

A dose de radiação efetiva da tomografia TCFC varia de acordo com a marca comercial do aparelho e com as especificações técnicas selecionadas durante a tomada (campo de visão, tempo de exposição, miliamperagem e quilovoltagem). Porém, de um modo geral, ela se mostra significantemente reduzida em comparação à tomografia computadorizada tradicional. Quando comparada às radiografias convencionais, a dose de radiação da TCFC apresenta-se similar à do exame periapical da boca toda, ou equivale aproximadamente a 4 a 15 vezes a dose de uma radiografia panorâmica. ${ }^{16,17}$

Os tomógrafos por feixe cônico disponível hoje no mercado possuem um mesmo princípio em seu funcionamento. Fazem um giro parcial ou total ao redor do objeto a ser escaneado, utilizando, em sua maioria, um feixe de raios $X$ de forma pulsátil, que atravessa e é atenuado pelo objeto até ser captado pelos sensores.
Apenas em algumas marcas comercias de tomógrafos de feixe cônico (Accuitomo, CB MercuRay, lluma Ultra Cone e Prexion 3D) o feixe de raios $X$ é contínuo, ao invés de pulsátil. As unidades atuais de feixe cônico podem ser divididas em dois grupos, com base no detector ou sensor de imagem: um intensificador de imagem combinado com dispositivos de acoplado de carga (IIT/CCD) ou um detector de imagens de tela plana (flat panel), sendo que o detector de tela plana cada vez mais vem sendo empregado nos aparelhos devido à melhor qualidade de imagem proporcionada e maior número debits. ${ }^{18}$

As imagens obtidas das múltiplas exposições geram um volume cilíndrico e o computador realiza a reconstrução primária. Depois, para trabalho, executamse reconstruções secundárias da imagem de acordo com as necessidades e com os protocolos de atendimento. Além dos cortes, podem-se gerar, a partir da TCFC, imagens planas ou tridimensionais da radiografia panorâmica em tamanho real 1:1, imagem da teleradiografia lateral, frontal, e muitos outros exames com uma nitidez muito maior do que os exames convencionais. ${ }^{18,19}$ As imagens digitais são formadas por pequenos pontos, a menor unidade destas, que são pequenos quadrados com medidas laterais idênticas, largura $(x)$ e altura $(\mathrm{y})$, sendo chamado de pixel (Picture Element). Como a tomografia é um volume tridimensional, um novo plano é adicionado, a profundidade ( $\mathrm{z}$ ), constituindo então não mais um quadrado e sim um cubo, chamado de voxel (Volume Element). A tomografia de feixe cônico possui voxel isométrico voxel com altura, largura e profundidade (de iguais dimensões) e voxel isomorfo, aumentado à capacidade de reproduzir detalhes dos tecidos duros com maior nitidez e clareza do que as tomografias médicas (que não possuem voxels isométricos), especialmente de estruturas delicadas, por exemplo, lâmina dura. Teoricamente, quanto 
menor o tamanho do voxel, tanto mais nítida tende ser a imagem, mas outros fatores como a qualidade do sensor, o projeto do aparelho, a estabilidade do paciente e o software interferem na nitidez final. ${ }^{18,19}$

Apesar das diversas vantagens citadas pelas imagens geradas pelos tomógrafos de feixe cônico, algumas limitações surgem durante a aquisição da imagem e são conceituadas como artefatos de imagem que são definidos como uma estrutura visualizada junto à imagem formada por meio dos dados usados na reconstrução que não está presente no objeto cuja tomada foi realizada. De modo geral, artefatos são induzidos por discrepâncias entre as reais condições físicas e a formação matemática utilizada para fazer a reconstrução em três dimensões. ${ }^{19,20}$

Há diferentes tipos de artefatos que podem estar presentes nas imagens tomográficas, sendo que cada um possui um fator determinante para o seu aparecimento. Beledelli e Souza ${ }^{11}$ analisaram os tipos de artefatos e os classificaram em: artefatos de movimento, artefatos gerados por materiais muito densos, artefatos de ruído, artefatos de espalhamento ou dispersão, artefato de extinção e artefatos de efeito do feixe cônico. É importante termos o conhecimento que o artefato de imagem pode ser gerado por diversas causas e é um fator prejudicial para a análise de imagens geradas por tomógrafos, pois podem levar a um diagnóstico equivocado.

Tohnak et al. ${ }^{12}$ relatam que a TCFC é de relevante importância para o diagnóstico, localização e reconstrução de imagens tomográficas com excelente precisão, auxiliando os profissionais da área da saúde no planejamento e tratamento dos pacientes.

Ozer, ${ }^{13}$ de acordo com a norma da Academia Europeia Dental e Radiologia Maxilofacial, diz que alguns parâmetros devem ser seguidos para evitar exposição excessiva da radiação ao paciente. A TCFC só deve ser indicada quando a radiografia convencional não for suficiente para o diagnóstico; devese escolher o menor tamanho possível de volume adquirido para fornecer uma menor dose ao paciente; e ainda escolher uma resolução de imagem adequada com menor dose alcançada para a doença em questão.

Hatcher ${ }^{14}$ afirma que para saber manipular os protocolos padrões da obtenção da imagem na TCFC, a fim de obter a otimização da técnica, é necessário ter o conhecimento de alguns parâmetros que podem interferir no resultado final da imagem. Durante a técnica, o operador necessita saber as variáveis contidas em um protocolo. Essas incluem o campo de visão (FOV); o tamanho do voxel; o tempo de escaneamento; os parâmetros de $\mathrm{mA}$ e a imobilização do paciente. A qualidade das imagens está relacionada com algumas características físicas como, volume parcial, escala dinâmica, modulação de transferência e sinal-ruído. Quando se tem um tempo de aquisição menor, a mA é reduzida, diminuindo a quantidade de fótons por voxel, que diminui o sinal, aumentando o ruído na imagem.

Melo et al, ${ }^{15}$ variando o tamanho do voxel, avaliarama capacidade de diagnóstico da TCFC em detectar fraturas radiculares longitudinais e a influência da presença de guta-percha e de núcleos metálicos. Foram utilizados 180 dentes divididos em três grupos experimentais: $\mathrm{Ae}, \mathrm{Be}$ e $\mathrm{Ce}$, no qual todos apresentavam fraturas induzidas artificialmente e três grupos controles: Ac, Bc e Cc. Os grupos Be e Bc foram preenchidos com cones de guta-percha e os grupos Ce e Cc receberam núcleos metálicos. Todos os dentes foram posicionados em um crânio seco e escaneados no tomógrafo i-CAT em duas resoluções de voxel (0,3 e 0,2 mm). Os resultados mostraram que a presença de guta-percha ou núcleo metálico reduziu a sensibilidade e especificidade em ambas as resoluções do voxel, mas sem diferença estatística, enquanto que os valores de 
sensibilidade foram superiores para o voxel de 0,2 mm. Diante disso, concluiu-se que a resolução de $0,3 \mathrm{~mm}$ não demonstrou ser um protocolo confiável para a visualização de fraturas longitudinais.

Ozer ${ }^{16}$ avaliou a capacidade de detecção de fraturas radiculares longitudinais em quatro diferentes resoluções de voxe/ na TCFC. Foram utilizados 60 dentes pré-molares extraídos de humanos, onde em metade da amostra fraturas longitudinais foram induzidas e após, os fragmentos foram reposicionados com cola e colocados em uma mandíbula para a aquisição das imagens no tomógrafo i-CAT (120 KvP, 5 mA e FOV: $4 \mathrm{~cm}$ ) em quatro resoluções: 0,125 $\mathrm{mm}$ de voxel (4 segundos para a aquisição); $0,2 \mathrm{~mm}$ de voxel (4 segundos para a aquisição); $0,3 \mathrm{~mm}$ de voxel (7 segundos para a aquisição); e 0,4 mm de voxel (7 segundos para a aquisição). As imagens foram avaliadas no programa XoranCAT para a presença e a ausência de fratura. Os resultados obtidos mostraram que não foi encontrada diferença na sensibilidade entre os quatro valores de voxel 0,125 mm (98\%), 0,2 $\mathrm{mm}(97 \%), 0,3 \mathrm{~mm}$ (93\%), 0,4 mm (91\%).

Loubele et al. ${ }^{17}$ compararam a qualidade de imagem e a dose de radiação de 4 TCFC (Accuitomo 3D, Mercury, Newtom 3G e i-CAT) e um tomógrafo computadorizado médico (Sensation 16). Os resultados mostraram que o i-CAT obteve a melhor qualidade de imagem com menor dose de radiação.

Kamburoglu et al. ${ }^{18}$ compararam as imagens radiográficas convencional; digital $C C D$ e PSP; e TCFC para detectar fratura radicular horizontal em dentes extraídos de humanos. Os fragmentos dentários, após a realização das fraturas, foram colados e posicionados dentro dos alvéolos de uma mandíbula seca para a realização das imagens. As imagens radiográficas foram adquiridas no aparelho Gendex Oralix, usando filme periapical convencional tipo E, sensores digitais CCD RVG
5.0 e placa de fósforo fotoestimulável (PSP) Digora Optime. As imagens de tomografia foram adquiridas no TCFC Accuitomo 3D (FOV: 3x4 $\mathrm{cm}$; voxel: 0,125 mm; $60 \mathrm{kVp}$ ). Os resultados mostraram que a sensibilidade da imagem de TCFC foi significantemente mais alta $(0,92)$ que as imagens de todas as radiografias intraorais em duas dimensões (Digora: 0,71; CCD: 0,68; Filme tipo $E: 0,74)$ e estas não apresentaram diferenças significantes entre si. Concluiu-se que as fraturas podem não ser detectadas devido aos aspectos anatômicos e à sobreposição de estruturas dentais adjacentes quando as técnicas radiográficas em bidimensionais são utilizadas.

Hassan et al. ${ }^{19}$ compararam a acurácia da TCFC e radiografia intraoral na detecção de fraturas radiculares longitudinais e avaliaram a influência do preenchimento do canal na visualização dessas fraturas. As imagens de 80 dentes foram obtidas no tomógrafo i-CAT (120 kVp; $6 \mathrm{~cm}$ de FOV, $5 \mathrm{~mA}$ e 0,25 mm de voxel) e no aparelho radiográfico intraoral da Siemens utilizando o sensor de placa de fósforo Digora em duas diferentes aquisições: Uma angulação ortorradial e uma mesio-angular. As imagens tomográficas foram avaliadas nos três planos de reconstrução axial, coronal e sagital. Os resultados mostraram que a sensibilidade e a acurácia da TCFC foram significantemente maiores que na radiografia intraoral para a detecção das linhas de fraturas. A presença de preenchimento do canal não reduziu a acurácia da TCFC, embora a especificidade tenha sido reduzida.

Costa el al. ${ }^{20}$ estudaram a precisão da TCFC com FOV menor para a detecção de fratura radicular horizontal com a presença e ausência de pino metálico. Quarenta dentes foram divididos em quatro grupos com base na presença de pinos metálicos e fratura radicular horizontal. Foi realizado o exame de TCFC nos dentes em resolução voxel de $0,2 \mathrm{~mm}$. A especificidade e a sensibilidade 
foram avaliadas e concluíram que ao reduzir o FOV ocorre um alto poder de varredura e maior precisão em detectar fratura radicular horizontal em raízes sem pino metálico, porém, quando presente o pino metálico, reduz significativamente a sensibilidade e a especificidade desse exame.

Akabane et al. ${ }^{21}$ realizaram um trabalho objetivando avaliar o emprego da tomografia computadorizada como opção para diagnosticar fraturas radiculares verticais. Foram utilizados dez dentes humanos extraídos que sofreram previamente fraturas radiculares verticais onde não houve separação dos fragmentos, e foram realizadas tomadas radiográficas periapicais, na técnica do paralelismo, e exames com tomografia computadorizada. Esses pesquisadores observaram que, na avaliação das radiografias periapicais, nenhuma fratura foi detectada. Os laudos tomográficos detectaram fraturas em todos os dentes avaliados. Concluíram que o exame tomográfico apresentou resultados confiáveis quanto à detecção de fraturas radiculares verticais, fato não observado no exame obtido por radiografias periapicais.

Mestka et al., ${ }^{22}$ em sua pesquisa, analisaram in vivo a acurácia de dois tomógrafos cone beam (Feixe cônico) para detectar fraturas radiculares verticais em dentes tratados endodonticamente. Trinta e nove dentes foram tratados endodonticamente com suspeita de fratura vertical radicular (FRV). Nenhuma linha de fratura era visível em radiografias periapicais. Foram usados dois aparelhos cone beam: NewTom 3G e o Accuitomo 3D 170. Três observadores avaliaram as imagens de TCFC de forma independente e tempos distintos. A sensibilidade, a especificidade e a precisão para o NewTom $3 G$ foram $75 \%, 56 \%$, e $68 \%$, respectivamente, e para o Accuitomo 3D 170 foram $100 \%, 80 \%$, e $93 \%$, respectivamente. Eles puderam concluir que a utilização do aparelho Accuitomo 3D 170 obteve maior especificidade e precisão para detectar fraturas radiculares verticais em dentes endodonticamente tratados comparado ao aparelho NewTom 3G. Eles também sugerem que a reprodutibilidade e a precisão para detectar FRV está diretamente relacionada ao sistema de TCFC utilizado.

Cohen et al..$^{23}$ avaliaram a aplicabilidade da TCFC no diagnóstico e plano de tratamento em casos de traumas. Relataram um caso clínico em que a radiografia periapical apresentou uma fratura radicular horizontal em um incisivo central superior esquerdo com separação de fragmentos e uma área radiolúcida na linha de fratura e sem patologia apical observada. As imagens da TCFC mostraram uma fratura radicular oblíqua na face palatina da raiz e também fratura do osso alveolar tanto nas faces palatina como vestibular. Concluíram que essas imagens foram fundamentais para o estabelecimento de um plano de tratamento adequado, assim como no acompanhamento do caso.

Para a visualização desses exames, existem diversos softwares específicos para essa função. Alguns com necessidade de adquirir chave de acesso, no qual existe um custo financeiro e outros com livre acesso.

O trabalho realizado por Wanzeler ${ }^{24}$ avaliou os programas de imagens odontológicas disponíveis gratuitamente na internet como ferramentas para a prática do cirurgião-dentista e que funcionam em computadores pessoais. Três programas foram selecionados de acordo com os critérios de inclusão e exclusão, eles são: InVivoDentalDemo, DentalSlice e CS $3 D$ Imaging Software. Foram analisadas as funções e ferramentas que são essenciais na prática diária do cirurgião-dentista radiologista, assim como para outras especialidades na área odontológica: criar cortes panorâmicos, transversais, axiais, sagitais, coronais e parasagital; reconstrução multiplanares (MPR); modelo de reconstrução 3D; identificação 
do canal mandibular no modelo 2D e 3D; simulação da posição do implante nos planos 2D e 3D; aplicação de medições de ângulos e distâncias; gerar em CD com imagens DICOM; linhas de referências; tornar dados dos exames anônimos e exportar imagens em JPG, BMP e DICOM. O autor concluiu na pesquisa que os programas avaliados tiveram resultados satisfatórios e disponibilizam as ferramentas necessárias para o profissional trabalhar. Testar e avaliar esses softwares é de grande importância para que todos tenham conhecimento, lembrando que, o ideal é analisar quais são as suas necessidades, e então escolher o melhor programa que se adapte a elas, sendo que se recomenda testar mais de um.

\section{Dıscussão}

O diagnóstico clínico e radiográfico de fraturas radiculares longitudinais em dentes tratados endodonticamente é difícil, e na maioria dos casos a extração dentária é o tratamento de escolha. ${ }^{7,25}$

Fraturas radiculares sem a separação de fragmentos podem não ser detectadas quando são utilizados métodos radiográficos bidimensionais, porque nessas técnicas radiográficas ocorre a sobreposição de estruturas e os artefatos podem simular ou esconder as linhas de fraturas. . $^{3,5,6}$

A sensibilidade dos exames tomográficos é maior do que as radiografias periapicais

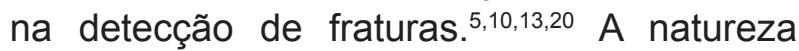
tridimensional da TCFC permite visualizar a linha de fratura em múltiplos ângulos e orientações diferentes em reconstruções de espessura fina. Já a especificidade é reduzida, pois cones de guta-percha e outros materiais hiperdensos podem criar artefatos em forma de raios de sol, imitando as linhas de fratura limitando o diagnóstico. ${ }^{10,15,27}$

O diagnóstico por imagens é uma parte indispensável na prática odontológica, tais exames envolvem a exposição de pacientes à radiação ionizante. $A$ filosofia de segurança da radiação utiliza o princípio ALARA (As Low As Reasonably Achievable), acrônimo para a expressão "tão baixo quanto razoavelmente exequível", como base para orientar todas as etapas do uso de radiação, os projetos de instalações dos equipamentos de irradiação e os procedimentos de proteção. 8,11,25

Embora haja fatores positivos no uso da TCFC, como obtenção de imagens de alta resolução e tridimensionalidade, o exame tomográfico apresenta doses de radiação e custos mais elevados do que as radiografias intraorais e panorâmicas convencionais, sendo assim é considerada somente quando técnicas radiográficas convencionais não são suficientes para o correto diagnóstico. ${ }^{2,10,15}$

Ainda como uma técnica imaginológica mais recente, apresenta interferência, ressaltase que objetos metálicos podem gerar artefatos indesejáveis nas imagens da tomografia, destacando-se restaurações metálicas, implantes osseointegrados, aparelhos ortodônticos, núcleos intrarradiculares e próteses fixas. ${ }^{20}$ Esse efeito chamado de "beam hardening" faz que a borda do objeto tenha a aparência mais brilhante do que o centro do objeto. Fatores como campo de visão, espessura do corte e dosagem do aparelho podem interferir no aumento ou na redução de artefatos metálicos nas imagens tomográficas. ${ }^{8,17,26}$ Portanto, além dos objetos que causam interferência, a qualidade de imagem também está diretamente relacionada à sensibilidade de cada aparelho de TCFC para detectar fraturas radiculares.

A visualização do exame em DICOM deixou de ser responsabilidade integral dos especialistas em radiologia com chaves de acesso restritas, o qual gera um custo financeiro para adquirir. O cirurgião-dentista, independente da sua especialidade, tem o 
conhecimento intelectual e outros recursos para adquirir programas, por exemplo, de forma gratuita.

\section{Conclusão}

Diversos estudos comprovaram que a TCFC é de grande aplicabilidade em endodontia, apesar dos artefatos que podem ser gerados, suas imagens fornecem maior grau de confiabilidade em relação às radiografias convencionais, auxiliando no diagnóstico e planejamento endodôntico, e é muito utilizada para a detecção de fraturas radiculares. Porém, apesar de superar as limitações das radiografias convencionais, a TCFC não deve ser usada rotineiramente, mas somente nos casos onde as radiografias convencionais forem consideradas inadequadas para o diagnóstico, comprometendo o tratamento.

\section{REFERÊNCIAS}

1. Hassan B, Metska ME, Ozok AR, Stelt PVD, Wesselink PR. Detection of vertical root fracture in endodontically treated teeth by a cone beam computed tomography scan. J. Endod., 2009; 35(5): 719-22.

2. American Association of Endodontists. Endodontics: Colleagues for Excellence Cracking the Cracked Tooth Code: Detection and Treatment of Various Longitudinal Tooth Fractures. American Association of Endodontists, 2008.

3. Azevedo B et al. Influence of the beam hardness on artifacts in Cone-Beam CT. Oral Surg. Oral Med .Oral Pathol. Oral Radiol Endod., 2008; 107: 1.124-30.

4. Cavalcanti, M. Tomografia computadorizada por feixe cônico. Interpretação e diagnóstico para o Cirurgião-Dentista, 1. ed., São Paulo: Editora Santos, 2010.

5. Scarfe WC, Farman AG, Sukovic P. Clinical Aplications of Cone-Beam Computed
Tomography in Dental Practice. J. Can. Dent. Assoc., 2006; 72: 75-80.

6. Schulze D., Heiland M., Blake F., Rother U., Schmelzle R. Evaluation of Quality of Reformatted images from Two Cone-beam Computed Tomography Systems. Journal of Cranio-Maxillofacial Surgery, 2005; 33: 19-23.

7. Bueno, MR, Estrela C., Azevedo BC, Brugnera Junior A., Azevedo JR. Tomografia computadorizada cone beam: revolução na Odontologia. Rev. Assoc. Paul. Cir. Dent., 2007; 61(5): 354-63.

8. Bernades RA, De Moraes IG, Húngaro Duarte MA, Azevedo BC, De Azevedo $\mathrm{JR}$, Bramante CM. Use of cone-beam volumetric tomography in the diagnosis of root fractures. Oral Surg. Oral Med. Oral Pathol. Oral Radiol. Endod., 2009; 7: 108270.

9. Haiter NF, et al. Diagnostic accuracy of cone beam computed tomography scans compared with intraoral image modalities for detection of caries lesions. Dentomaxillofac. Radiol., 2008; 37:18-22.

10. Hassan B., Metska ME, Ozok AR, Stelt PVD, Wesselink PR. Detection of vertical root fracture in endodontically treated teeth by a cone beam computed tomography scan. J. Endod. 2009; 35(5): 719-22.

11. Beledelli R., Souza, CHP. O que são e como se formam os artefatos nas imagens da Tomografia Computadorizada de Feixe Cônico. ver. ABRO, 2012, 13 (1): 2-15.

12. Tohnak S. et. al. Dental CT metal artifact reduction based on sequential substitution. Dentomaxilofacial Radiology, 2011; 40: 184-190.

13. Ozer SY. Detection of Vertical Root Fractures by Using Cone Beam Computed Tomography with Variable Voxel Sizes in an In Vitro Model. J. Endod., 2011; 37: 75-79.

14. Hatcher DC. Operational Principles for Cone-beam Computed Tomography. J. Am. Dent. Assoc., 2010; 141: 3S-6S.

15. Melo SLS, Bortoluzzi EA, Abreu M., Corrêa LR, Corrêa M. Diagnostic Ability of a ConeBeam Computed Tomography Scan to 
Assess Longitudinal Root Fractures in Prosthetically Treated Teeth. J. Endod., 2010; 36: 1.879-82.

16. Ozer SY. Detection of Vertical Root Fractures of Different Thicknesses in Endodontically Enlarged Teeth by Cone Beam Computed Tomography versus Digital Radiography. J. Endod., 2010; 36: 245-259.

17. Loubele M., Guerrero ME, Jacobs R., Suetens P., van Steenberghe D. A Comparison of jaw dimensional and quality assessments of bone characteristics with cone-beam CT, spiral tomography, and multi-slice spiral CT. Int. J. Oral Maxillofac. Implants, 2007; 22(3): 446-454.

18. Kamburoglu K., Cebeci ARI, Grongahl HG. Effectiveness of Limited Cone-beam Computed Tomography in the Detection of Horizontal Root Fracture. Dental Tramautology, 2009; 25: 256-261.

19. Hassan B., Metska ME, OzokAR, Stelt PVD, Wesselink PR. Comparison of Five Cone Beam Computed Tomography Systems for the Detection of Vertical Root Fractures. J. Endod., 2010; 1: 126-9.

20. Costa FF, Gaia BF, Umestsubo OS, Cavalcanti MG. Detection of horizontal root fracture with small-volume conebeamcomputedtomographyinthepresenceand absence of intracanal metallic post. J. Endod., 2011; 37(10): 1.456-9.

21. Akabane CE, Fukunaga D., Baratella T., Camargo SCC, Mancini R., Shimabuko DM. Comparação entre radiografia periapical e tomografia computadorizada no diagnóstico de fraturas radiculares verticais. Revista Brasileira de Pesquisa em Saúde, 2010; 12(2): 61-68.

22. Metska ME, Aartman IH, Wesselink PR, Özok AR. Detection of vertical root fractures in vivo in endodontically treated teeth by cone-beam computed tomographyscans. J. Endod., 2012; 38 (10): 1.344-7.

23. Cohen S., Blanco L., Berman L. Vertical Root Fractures Clinical and Radiographic Diangosis. JADA, 2003; 134: 434-41.

24. Wanzeler, AMV. Análise dos softwares gratuitos para tomografia computadorizada de feixe cônico de interesse aos cirurgiõesdentistas. Rev. Bras. Odontol., 2015; 72: 51-5.

25. Vidigal BML; Abreu SGA; Silva FA; Moreira GP; Manzi FR. Uso da Tomografia Cone Beam na Avaliação de Fraturas Radiculares. Rev. Bras. Odontol., 2014; 71 (2): 152-5.

26. Cardoso CBM;Benetti F.; FerreiraLL; DezanJúnior E.; Gomes-Filho JE; Cintra LTA. Emprego da Tomografia Computadorizada Cone-beam em Endodontia: Revisão de Literatura. Rev. Odontol. UNESP, 2014; 43: 245.

27. Lima TFR, Souza-Filho FJ, Soares AJ. Tomografia Computadorizada de Feixe Cônico como Método Auxiliar na Localização de Canais Calcificados. Dental. Press. Endod., 2014; 4(2): 74-78.

Submetido em: 22-8-2015

Aceito em: 26-4-2016 